\title{
MODELAGEM MATEMÁTICA DAS CINÉTICAS DE EXTRAÇÃO SUPERCRÍTICA DE PRODUTOS NATURAIS
}

\author{
A. P. S. SILVA ${ }^{1}$, R. M. CORDEIRO ${ }^{2}$, C. C. R. BATISTA ${ }^{1}$, J. R. S. BOTELHO ${ }^{1}$, J. E. R. \\ SILVA $^{1}$, M. E. ARAÚJO ${ }^{1}$, R. N. CARVALHO Jr ${ }^{1}$. \\ ${ }^{1}$ Universidade Federal do Pará \\ ${ }^{2}$ Universidade Federal do Pará, Instituto Tecnológico, Departamento de Engenharia Química \\ Email para contato: anapaula-eng@hotmail.com
}

\begin{abstract}
RESUMO - O objetivo deste trabalho é determinar parâmetros de transferência de massa a partir das cinéticas de extração dos óleos de sementes de maracujá (Passiflora edulis) e polpa de pupunha (Guilielma speciosa), através da avaliação dos modelos matemáticos de Tan e Liou (1989), Goto et al. (1993) e Martínez et al.(2003). As cinéticas foram realizadas utilizando-se $400 \mathrm{~g}$ de sementes do maracujá e $20 \mathrm{~g}$ de polpa da pupunha nas condições de $250 \mathrm{Bar}$ e $50^{\circ} \mathrm{C}$ para pupunha e 250 Bar e $70^{\circ} \mathrm{C}$ para o maracujá e diferentes configurações de leito. Consequentemente, foi elaborada uma base de dados com os resultados obtidos através da comparação e avaliação das previsões dos modelos aos dados experimentais. Os modelos foram analisados de acordo com os erro relativo $(e)$, erro médio relativo $(E m)$, desvio padrão $(S)$ e faixa de erro $(R)$. O modelo de Martínez et. al.(2003) foi o que apresentou os melhores ajustes para o maracujá e para a pupunha, os modelos de Tan e Liou (1989) e Martínez et al.(2003) apresentaram melhores resultados.
\end{abstract}

\section{INTRODUÇÃO}

A região amazônica possui uma vasta diversidade de plantas e animais, e com isso, vem recebendo especial atenção pelas indústrias alimentícias, principalmente por conta de suas plantas, que possuem alto poder antioxidante e excelente valor nutritivo.

A pupunha (Guilielma speciosa) é um fruto rico em proteínas, carboidratos, e vários elementos minerais, como cálcio, ferro, fósforo, entre outros. Também se destaca como fonte rica em pró-vitamina A. (CAVALCANTE, 1991.). Possui elevado conteúdo de óleo e grande valor nutritivo, essas características podem fazer do fruto da pupunheira, um produto com imenso valor de mercado e de grande valor na segurança alimentar (ZUMBADO e MURILLO, 1984).

O maracujá (Passiflora edulis) é um fruto cultivado em diversas regiões do mundo. E apesar de não se ter comprovação de sua natividade, acredita-se que o fruto seja nativo do Brasil. Seu cultivo se dá em larga escala no Brasil, através da produção de sucos. Cerca de 60 espécies produzem frutas comestíveis, duas destas, são cultivadas em todo o mundo e podem ser usadas, direta ou indiretamente como alimento, ou para obtenção de doces, licores, refrescos, sorvetes, sucos e em misturas com bebidas alcoólicas. Atribui-se ao maracujá 
propriedades médico-farmacêuticas tais como: ação anti-helmíntica e sedativa. (CORRÊA, 1994).

A utilização de compostos sintéticos nos produtos industrializados vem sofrendo cada vez mais rejeições por parte das indústrias tanto alimentícia quanto farmacêutica. Desta forma, a comunidade científica tem buscado alternativas de melhoria na obtenção de compostos naturais. O problema da extração com outros métodos convencionais, como o solvente orgânico, é que além de deixar resíduos frequentemente proibidos em alimentos, apresenta o problema da transformação oxidativa que o extrato sofre quando o solvente é eliminado. (SEBASTIÁN et. al., 1998).

Por ser uma tecnologia limpa, a Extração com fluido supercrítico (SFE, Supercritical fluid extraction) vem sendo difundida nas mais diversas áreas industriais, dadas às suas vantagens únicas tais como temperaturas baixas, extração seletiva, simplicidade e a recuperação do produto livre do solvente.

O maior obstáculo ao uso da tecnologia supercrítica na indústria é o alto investimento necessário para a instalação de uma unidade de extração. A minimização do custo é possível através da otimização do processo, que envolve o dimensionamento do equipamento e a definição das condições operacionais. Para a otimização destas condições, é de fundamental importância a modelagem matemática dos processos de extração e separação. A análise de uma curva de extração para um determinado processo pode ajudar a definir o volume do extrator, a vazão requerida de solvente para a taxa de extração desejada, entre outros. Para tanto, os modelos matemáticos devem ajustar parâmetros cujo valor possa ser predito como função das condições operacionais, de forma que seja possível a partir de curvas modeladas projetar um processo de extração supercrítica em escala industrial. (MARTÍNEZ, 2005)

O objetivo deste trabalho consiste na determinação de parâmetros de transferência de massa aplicados à SFE a partir do tratamento de dados cinéticos obtidos com a extração de dois diferentes extratos vegetais através da utilização de ferramentas computacionais. Consequentemente, elaborar um banco de dados destes parâmetros, possibilitando a utilização destes para futuros estudos de aumento de escala de produção e servindo de base para as indústrias que utilizam essas matérias-primas em seus processos produtivos.

\section{METODOLOGIA}

\subsection{Extração Supercrítica}

Curvas globais de extração: As curvas globais de extração do maracujá foram obtidas por Corrêa (1994) e da pupunha por Araújo et. al.(2000), conforme descrito nas seguintes etapas: a) para obtenção das curvas globais do maracujá (Passiflora edulis) foram utilizadas sementes da mesma, fornecidas pela AMAFRUTAS (Benevides - Pará - Brasil). O diagrama esquemático da unidade de extração está descrito em Corrêa (1994). Foram utilizadas 400g de sementes de maracujá. As condições de extração foram $70^{\circ} \mathrm{C}$ e 250 bar com vazão de $\mathrm{CO}_{2}$ de $0,025 \mathrm{Kg} / \mathrm{s}$. O leito fixo apresentou as seguintes características físicas: densidade real 929,7 $\mathrm{Kg} / \mathrm{m}^{3}$, densidade aparente $744,7 \mathrm{Kg} / \mathrm{m}^{3}$, diâmetro médio das partículas $0,000319 \mathrm{~m}$, porosidade do leito 0,2 e volume do leito $0,0035 \mathrm{~m}^{3}$. b) para obtenção das curvas globais da pupunha 
(Guilielma speciosa) foram utilizadas $20 \mathrm{~g}$ de polpa adquiridas em lojas locais de Belém (ParáBrasil). O diagrama esquemático da unidade de extração utilizada está descrito em Araújo et. al. (2000). As condições de operação foram de $50^{\circ} \mathrm{C}$ e $250 \mathrm{bar}$ com vazão de $\mathrm{CO}_{2}$ de $0,31 \mathrm{Kg} / \mathrm{s}$. As configurações do leito fixo foram as seguintes: densidade real $960 \mathrm{Kg} / \mathrm{m}^{3}$, densidade aparente $10 \mathrm{Kg} / \mathrm{m}^{3}$, diâmetro médio das partículas $0,0004745 \mathrm{~m}$, porosidade do leito 0,99 e volume do leito $0,002 \mathrm{~m}^{3}$. Estes resultados foram utilizados como dados de entrada para os modelos da literatura.

\subsection{Modelagem Matemática}

Os dados experimentais (massa de extrato versus tempo de extração) das condições operacionais de $250 \mathrm{bar}, 70^{\circ} \mathrm{C}$, vazão de $\mathrm{CO}_{2}$ de $0,025 \mathrm{Kg} / \mathrm{s}$ do maracujá e 250 bar, $50^{\circ} \mathrm{C}$, vazão de $\mathrm{CO}_{2}$ de $0,31 \mathrm{Kg} / \mathrm{s}$ da pupunha com suas respectivas configurações de leito, foram utilizados para o ajuste dos parâmetros de processo utilizando os modelos de Goto et. al. (1993), Tan e Liou (1989) e Martínez et. al. (2003). Na tabela 1, encontram-se as equações dos modelos utilizados.

Tabela 1 - Equações dos modelos

\begin{tabular}{c|l}
\hline Modelo & Equação \\
\hline $\begin{array}{c}\text { Goto } \text { et. } \boldsymbol{A l} . \\
(\mathbf{1 9 9 3 )}\end{array}$ & $m=\frac{A\left[\frac{\beta}{K}+(1-\beta)\right] X_{0} \rho_{s} Q_{C O_{2}}}{\rho}\left\{\frac{\tau}{a_{1}}\left[\exp \left(a_{1} \frac{t}{\tau}\right)-1\right]+\frac{\tau}{a_{2}}\left[1-\exp \left(a_{2} \frac{t}{\tau}\right)\right]\right\}$ \\
$\begin{array}{c}\text { Tan e Liou } \\
(\mathbf{1 9 8 9 )}\end{array}$ & $m=\frac{A}{K_{d}}\left[1-\exp \left(K_{d} B\right)\right]\left[\exp \left(-K_{d} t\right)-1\right]$ \\
$\begin{array}{c}\text { Martínez et. } \\
\text { Al. (2003) }\end{array}$ & $m=\frac{X_{0} F_{0}}{\exp \left(b_{i} t_{m i}\right)}\left\{\frac{1+\exp \left(b_{i} t_{m i}\right)}{1+\exp \left[b_{i}\left(t_{m i}-t\right)\right]}-1\right\}$ \\
\hline
\end{tabular}

Foram utilizados os seguintes parâmetros estatísticos para a análise dos resultados da modelagem matemática: erro relativo $(e)$, erro médio relativo $(E m)$, desvio padrão $(S)$, faixa de erro $(R)$ expressos nas equações de 1 a 4 , respectivamente.

$$
\begin{aligned}
& e=\frac{m_{c a l}-m_{\text {exp }}}{m_{\text {exp }}} \\
& E_{m}=\frac{1}{N} \sum_{i} e \\
& S=\left[\frac{1}{N-1} \sum_{i}\left(e-E_{m}\right)^{2}\right]^{1 / 2} \\
& R=e_{\text {max }}-e_{\text {min }}
\end{aligned}
$$

\section{RESULTADOS E DISCUSSÃO}

Os parâmetros de processo referentes aos leitos fixos das sementes de maracujá e polpa da pupunha, necessários para o ajuste de parâmetros dos modelos de transferência de massa de Goto et. al. (1993), Tan e Liou (1989) e Martínez et. al. (2003) estão apresentados na tabela 2. 
Na tabela 3 estão apresentados os valores dos parâmetros dos modelos ajustados as curvas de cinética de extração.

Tabela 2 - Parâmetros de processo para a extração de óleo das matrizes vegetais

\begin{tabular}{ccc}
\hline Parâmetro & Caracujá & Condições \\
\hline $\mathbf{( 4 0 0 g})$ & Pupunha \\
$\mathbf{T}\left({ }^{\circ} \mathbf{C}\right)$ & 70 & $(\mathbf{2 0 g})$ \\
$\mathbf{P}(\mathbf{b a r})$ & 250 & 50 \\
$\mathbf{A ~ ( \mathbf { m } ^ { 2 } )}$ & 0,069 & 250 \\
$\mathbf{V}\left(\mathbf{m}^{\mathbf{3}}\right)$ & 0,0035 & 0,000000176833 \\
$\boldsymbol{Q}_{\boldsymbol{C o}}(\mathbf{K g} / \mathbf{s})$ & 0,025 & 0,002 \\
$\mathbf{H}(\mathbf{m})$ & 0,275 & 0,31 \\
$\mathbf{D}(\mathbf{m})$ & 0,08 & 0,044 \\
$\boldsymbol{\rho}_{\boldsymbol{s}}\left(\mathbf{K g} / \mathbf{m}^{\mathbf{3}}\right)$ & 929,7 & 0,029 \\
$\boldsymbol{\rho}_{\boldsymbol{C} \boldsymbol{o}_{\mathbf{2}}}\left(\mathbf{K g} / \mathbf{m}^{\mathbf{3}}\right)$ & 811,18 & 960 \\
$\boldsymbol{\varepsilon}(-)$ & 0,2 & 0,99 \\
\hline
\end{tabular}

Tabela 3 - Parâmetros dos modelos de transferência de massa

\section{Goto et. al. (1993) Tan e Liou Martínez et. al.}

(1989)

\begin{tabular}{cccccc}
\hline & $\boldsymbol{\Phi}$ & $\mathbf{K}$ & $\boldsymbol{K}_{\boldsymbol{d}}\left(\mathbf{m i n}^{-\mathbf{1}}\right)$ & $\mathbf{b i}\left(\mathbf{m i n}^{-\mathbf{1}}\right)$ & $\mathbf{t}_{\mathbf{m i}}(\mathbf{m i n})$ \\
Maracujá & 229,46 & 7,31 & $8,08256 \mathrm{E}-5$ & $4,8793 \mathrm{E}-4$ & 2871,20 \\
Pupunha & 2172,42 & 0,93 & $5,28142 \mathrm{E}-4$ & $4,30564 \mathrm{E}-4$ & $-70813,01$ \\
\hline
\end{tabular}

Nas tabelas 4 e 5 estão descritos os parâmetros estatísticos determinados a partir dos valores calculados, com os respectivos parâmetros ajustáveis, das curvas de cinética de extração dos óleos de maracujá e pupunha empregando $\mathrm{CO}_{2}$ supercrítico. Os modelos de Tan e Liou (1989), Martínez et al.(2003) e Goto et al. (1993) ajustaram quantitativamente as cinéticas de extração, apresentando baixos erros médios relativos.

O modelo de Martínez et al. (2003) apresentou os menores erros médios relativos e desvios padrão, para a cinética de extração do maracujá. O modelo de Tan e Liou (1989) apresentou o menor desvio padrão e o de Martínez et al.(2003) apresentou o menor erro médio relativo para a cinética de extração da pupunha.

Tabela 4 - Parâmetros estatísticos da curva de extração do óleo de maracujá

\section{Modelos}

\section{Erro Médio}

\section{Parâmetros Estatísticos}

Faixa de Erro

Desvio Padrão

Tan e Liou (1989)

$\begin{array}{lll}0,0909 & 1,0420 & 0,2462 \\ 0,0025 & 0,2592 & 0,0483 \\ 0,0382 & 0,8437 & 0,1906\end{array}$

Goto et al. (1993) 0,0382 0,8437 0,1906 
Tabela 5 - Parâmetros estatísticos da curva de extração do óleo de pupunha

\section{Modelos}

Parâmetros Estatísticos

Erro Médio Faixa de Erro Desvio Padrão

$\begin{array}{llll}\text { Tan e Liou (1989) } & -0,0174 & 0,3471 & 0,0887 \\ \text { Martínez et al.(2003) } & -0,0212 & 0,3303 & 0,0922 \\ \text { Goto et al. } \mathbf{( 1 9 9 3 )} & -0,0073 & 0,3443 & 0,0895\end{array}$

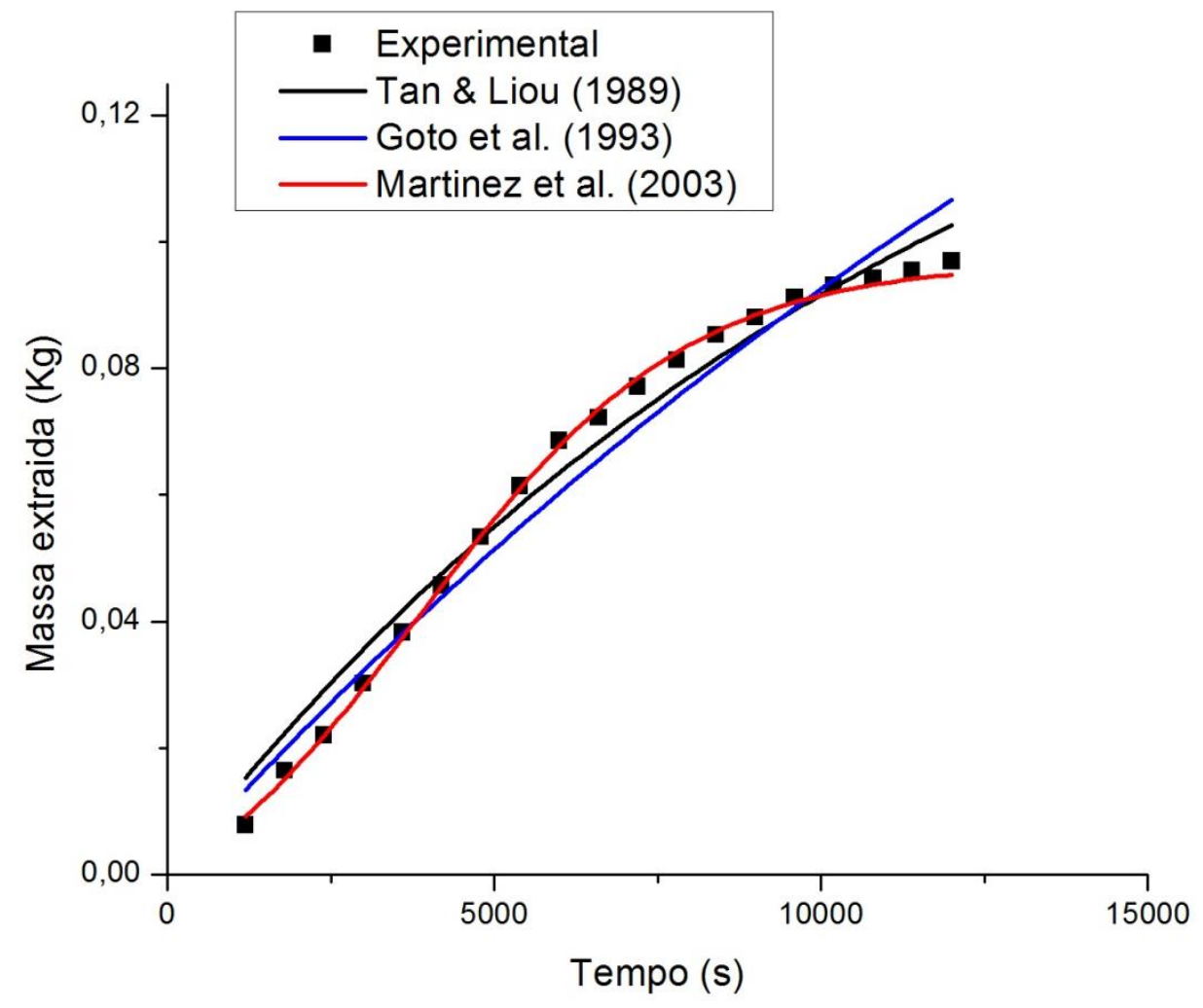

Figura 1 - Modelagem das cinéticas de extração do óleo de sementes de maracujá. 


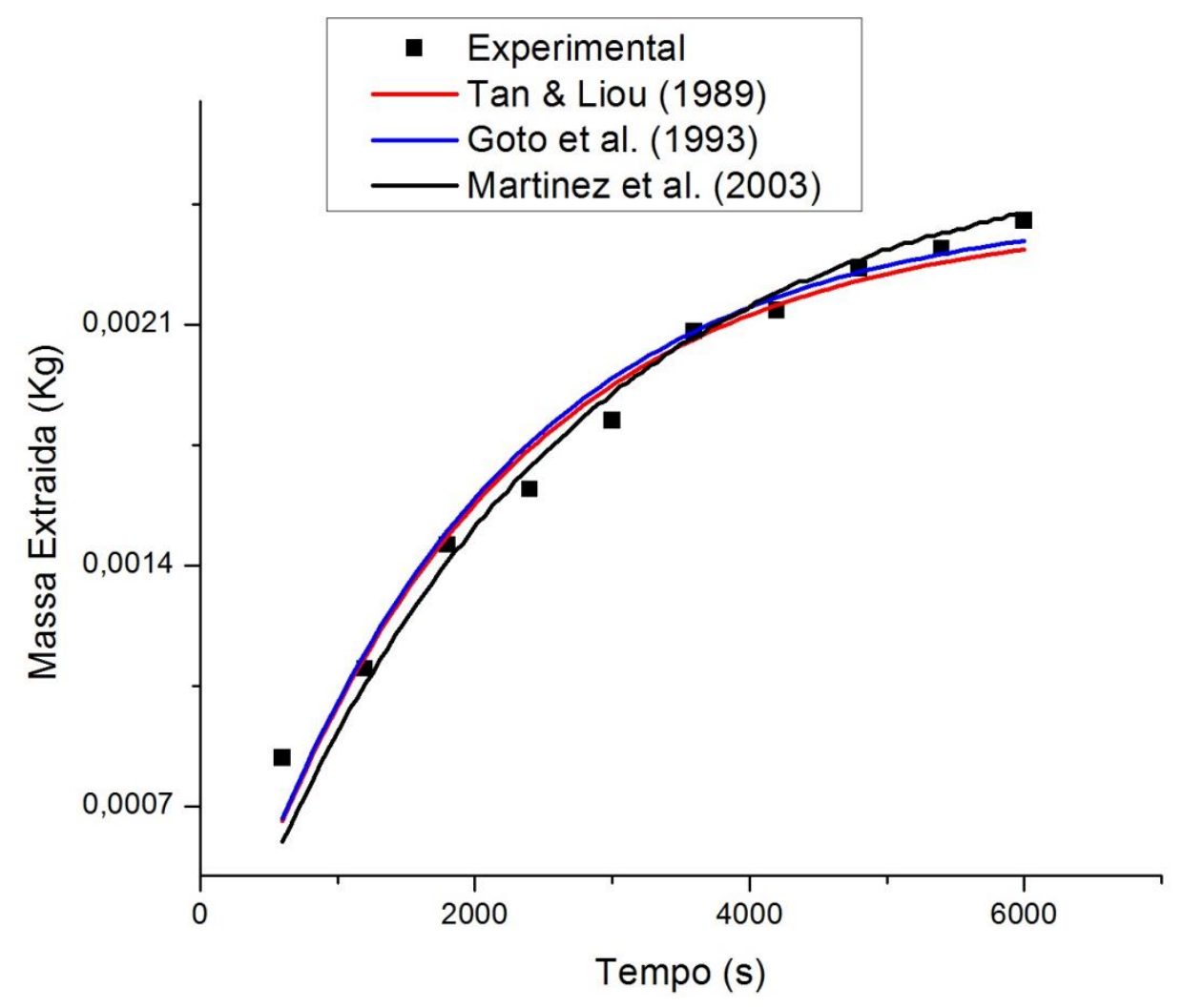

Figura 2 - Modelagem das cinéticas de extração do óleo da polpa da pupunha.

\section{CONCLUSÕES}

Conclui-se neste trabalho que todos os modelos de transferência de massa utilizados para modelagem das curvas globais de extração dos extratos de Maracujá e Pupunha descreveram satisfatoriamente as cinéticas de extração apresentando baixos desvios médios. Para o maracujá, o modelo de Martínez et.al. (2003) apresentou menores erros médios relativos e desvios padrão. Para a pupunha, os modelos de Tan e Liou (1989) apresentou o menor desvio padrão e o de Martínez et. al.(2003) apresentou o menor erro médio relativo.

\section{REFERENCIAS}

ARAÚJO, M. E.; MACHADO, N. T.; FRANÇA, L. F.; MEIRELES, M. A. A. Supercritical Extraction of Pupunha (Guilielma speciosa) oil in a fixed bed using carbono dioxide. Braz. J. of Chem. Eng., v. 17, n. 3, 2000.

CAVAlCAnTE, P. B., Frutas Comestíveis da Amazônia, Edições CEJUP, Museu Paraense Emílio Goeldi, 1991.

CORRÊA, N. C. F. Estudo da Cinética de Extração de Óleo da Semente de Maracujá com $\mathrm{CO}_{2}$ Supercrítico. Dissertação (Mestrado em Engenharia Química). Universidade Federal do Pará, Belém, 1994. 
GOTO, M.; SATO, M.; HIROSE, T. Extraction of Peppermint Oil by Supercritical Carbon Dioxide. J. of Chem. Eng. of Japan. n. 26, p. 401 - 407, 1993.

MARTÍNEZ, J. Extração de Óleos Voláteis e Outros Compostos com CO2 Supercrítico: Desenvolvimento de uma Metodologia de Aumento de Escala a partir da Modelagem Matemática do Processo e Avaliação dos Extratos Obtidos. Tese (Doutorado em Engenharia de Alimentos). Universidade Estadual de Campinas. Campinas, 2005.

MARTÍNEZ, J.; MONTEIRO, A. R.; ROSA, P. T. V.; MARQUES, M. O. M.; MEIRELES, M. A. A. Multicomponent Model to Describe Extraction of Ginger Oleoresin with Supercritical Carbon Dioxide. Industrial and Engineering Chemical Research, n. 42, p. 1057 - 1063, 2003.

SEBASTIÁN, S. L.; RAMOS, E.; IBÁÑEZ, E.; BUENO, J. M.; BALLESTER, L.; TABERA, J.; REGLERO, G. Dearomatization of Antioxidant Rosemary Extracts by Treatment with Supercritical Carbon Dioxide. J. of Agric. and Food Chem., n. 46, p. 13, 1998.

TAN, C.; LIOU, D. Modeling of Desorption at Supercritical Conditions. Am. Inst. of Chem. Eng. J., v. 6, n. 35, p. 1029 - 1031,1989.

ZUMBADO, M.; MURILLO, M. Composition and nutritive value of pejibaye (Bactris gasipaes) in animal feeds (Composição e valor nutritivo de pupunha em ração animal). Revista de Biología Tropical, v. 32, p. 51-56, 1984. 